\begin{tabular}{|l|l|l|}
\hline \multicolumn{2}{|c|}{ PublisherInfo } \\
\hline \hline PublisherName & $:$ & BioMed Central \\
\hline \hline PublisherLocation & $:$ & London \\
\hline \hline PublisherImprintName & $:$ & BioMed Central \\
\hline \hline
\end{tabular}

\title{
Articles selected in February 2004
}

\begin{tabular}{||l|l|l||}
\hline \multicolumn{2}{|c||}{ ArticleInfo } \\
\hline \hline ArticleID & $:$ & 771 \\
\hline \hline ArticleDOI & $:$ & $10.1186 /$ bcr769 \\
\hline \hline ArticleCitationID & $:$ & E12 \\
\hline \hline ArticleSequenceNumber & $:$ & 4 \\
\hline \hline ArticleCategory & $:$ & Article selection \\
\hline ArticleFirstPage & $:$ & 1 \\
\hline \hline ArticleLastPage & $:$ & 3 \\
\hline \hline & $:$ & RegistrationDate : 2004-3-3 \\
ArticleHistory & $:$ & OnlineDate \\
\hline \hline ArticleCopyright & $:$ & The Author(s)2004-3-3 \\
\hline \hline ArticleGrants & $:$ & \\
\hline \hline ArticleContext & $:$ & 130586633 \\
\hline \hline
\end{tabular}


Valerie Speirs, Aff1

Corresponding Affiliation: Aff1

Email: v.speirs@leeds.ac.uk

Aff1 Molecular Medicine Unit, University of Leeds, St James's University

Hospital, Leeds LS9 7TF, UK

\section{Articles selected from PubMed}

\section{References}

1. Wang LH, Yang XY, Zhang X, Mihalic K, Fan YX, Xiao W, Howard OM, Appella E, Maynard AT, Farrar WL: Suppression of breast cancer by chemical modulation of vulnerable zinc fingers in estrogen receptor. Nat Med. 2004, 10: 40-47.

2. Santen RJ, Song RX, Zhang Z, Yue W, Kumar R: Adaptive hypersensitivity to estrogen: mechanism for sequential responses to hormonal therapy in breast cancer. Clin Cancer Res. 2004, 10: 337-345.

3. Nicholson RI, Hutcheson IR, Knowlden JM, Jones HE, Harper ME, Jordan N, Hiscox SE, Barrow D, Gee JM: Nonendocrine pathways and endocrine resistance: observations with antiestrogens and signal transduction inhibitors in combination. Clin Cancer Res. 2004, 10: 346-354.

4. Strom A, Hartman J, Foster JS, Kietz S, Wimalasena J, Gustafsson JA: Estrogen receptor beta inhibits 17beta-estradiol-stimulated proliferation of the breast cancer cell line T47D. Proc Natl Acad Sci USA. 2004, 101: 1566-1571.

5. Hoffmann J, Bohlmann R, Heinrich N, Hofmeister H, Kroll J, Kunzer H, Lichtner RB, Nishino Y, Parczyk K, Sauer G, Gieschen H, Ulbrich HF, Schneider MR: Characterization of New Estrogen Receptor Destabilizing Compounds: Effects on Estrogen-Sensitive and Tamoxifen-Resistant Breast Cancer. J Natl Cancer Inst. 2004, 96: 210-218. 
6. Holmberg L, Anderson H, for the HABITS steering and data monitoring committees: HABITS (hormonal replacement therapy after breast cancer - is it safe?), a randomised comparison: trial stopped. The Lancet. 2004, 363: 453-455.

7. Balasenthil S, Sahin AA, Barnes CJ, Wang RA, Pestell RG, Vadlamudi RK, Kumar R: p21-activated Kinase-1 Signaling Mediates Cyclin D1 Expression in Mammary Epithelial and Cancer Cells. J Biol Chem. 2004, 279: 1422-1428.

8. Reiter R, Oh AS, Wellstein A, Riegel AT: Impact of the nuclear receptor coactivator AIB1 isoform AIB1-Delta 3 on estrogenic ligands with different intrinsic activity. Oncogene. 2004, 23: 403-409.

This PDF file was created after publication. 